\title{
Administración pública y tercer sector. Propuesta analítica y estudio del caso de Andalucía ${ }^{1}$
}

\author{
Clemente J. Navarro Yáñez \\ María Jesús Rodríguez García \\ Universidad Pablo de Olavide. Centro de Sociología Política \\ cnavyan@dts.upo.es \\ mjrodgar@dts.upo.es
}

\section{Resumen}

Suele argumentarse que la participación de entidades sin ánimo de lucro en la provisión de servicios públicos puede ser una vía para hacer frente a la crisis del Estado del bienestar. No obstante, son pocos los análisis que se centran en el estudio de la calidad de las relaciones que para ello establecen entidades del tercer sector y las agencias gubernamentales. Este artículo presenta una propuesta analítica y la aplica a un estudio de caso, partiendo del supuesto de que la calidad de tales relaciones depende de la información que las agencias gubernamentales poseen de las entidades con las que interactúan.

Palabras clave: agencias gubernamentales, tercer sector, calidad del pluralismo del bienestar, provisión de servicios.

\section{Abstract. Public Administration and Non-Profit Sector. Analitical proposal and study of Andalucia case}

It is a commonplace the argument about the benefits of non-profit inclusion in the provision of public services to reduce the welfare state crisis. Therefore, there are many few studies about the quality of relationships between non-profit sector and governmental agencies. This article presents an analytical proposal and its application by a case study. The main argument is that the quality of relations between non-profit sector and government depends of governmental agencies' information about non profit entities.

Key words: governmental agencies, non-profit sector, welfare-pluralism quality, services suply.

1. Este trabajo presenta algunos resultados del proyecto «Las entidades sin fin de lucro en Andalucía» realizado en 2000 con la financiación del Instituto Andaluz de Estadística (Programa de Fomento de la Investigación). El investigador principal fue Fernando Aguiar (IESA/CSIC), y colaboraron Manuel Pérez Yruela y Manuel Trujillo (IESA/CSIC), Alicia García (Universidad de Granada), además de Antonia Ramírez y Raquel Pastor (Universidad Pablo de Olavide). A todos ellos, muchas gracias. Las conclusiones del proyecto pueden consultarse en el documento de trabajo del IEA Aguiar y otros (2001). Una primera versión del texto fue presentada por los autores en las Jornadas sobre Economía Social y Relaciones Laborales (Sevilla, 28-29 de septiembre de 2000). Los autores agradecen a los evaluadores anónimos sus inestimables sugerencias que han mejorado sustantivamente la anterior versión del artículo. 


\section{Sumario}

1. Pluralismo del bienestar y tercer sector: sobre la necesaria aclaración de conceptos

2. Pluralismo del bienestar como problema de información

3. El análisis de los registros de entidades de las agencias gubernamentales
4. A modo de conclusiones: estilos políticos de interacción y calidad del pluralismo del bienestar

Bibliografía

No cabe duda que el denominado «tercer sector» viene jugando un papel cada vez más importante en las sociedades contemporáneas, ya sea actuando como provisor de servicios, ya sea reclamando cambios en la agenda de actuación de los poderes públicos o, incluso, promoviendo la incorporación de nuevos temas. Cuestiones, ambas, que suponen un continuo y creciente proceso de interacción con el estado y las administraciones públicas, con el objeto de mejorar el diseño de las políticas públicas y la provisión de servicios. De ello se deriva que la calidad de las relaciones entre ambos actores es un elemento básico en el desarrollo de los nuevos modelos de provisión de servicios públicos que incorporan a agentes no estatales.

Este artículo presenta una aproximación analítica a tal asunto concibiendo las relaciones entre estado y tercer sector como un problema de información, más concretamente, sobre aquélla que tienen las agencias gubernamentales de las entidades sin ánimo de lucro con las que colaboran. Posteriormente, se aplica empíricamente tomando como caso la comunidad autónoma de Andalucía.

\section{Pluralismo del bienestar y tercer sector: sobre la necesaria aclaración de conceptos}

Después de décadas señalando la crisis del Estado del bienestar, ya sea por la sobrecarga de demandas, por sus rigideces burocráticas o por sus problemas fiscales, las ciencias sociales se han empeñado en los noventa en analizar posibles soluciones. Básicamente, se señala la necesidad de crear nuevas formas de interacción entre estado y sociedad civil, una new governance caracterizada por la intensificación de las relaciones entre ambas esferas, e incluso, la participación de actores de la segunda en la provisión de servicios públicos (Kooiman, 1993; Mayntz, 1999). Y no se trata únicamente de un empeño analítico, sino de una realidad empírica presente en la provisión cotidiana de servicios ${ }^{2}$.

En el marco de esta tendencia, se señala la posible transición desde el Estado del bienestar al pluralismo del bienestar, o welfare mix, modelo de provisión de servicios públicos de bienestar en el que se incorporan como agentes ofe-

2. En este caso, los gobiernos locales se vienen mostrando bastante innovadores. Para un análisis empírico de tales tendencias en los gobiernos municipales de diversos países europeos y Estados Unidos, puede consultarse Clarke (1989). 
rentes el mercado, el sector informal (la familia) y el sector voluntario o tercer sector (entidades sin fin de lucro) (Rodríguez Cabrero, 1994). No obstante, entre ellos, ha sido el último el que ha despertado mayor interés, por considerarse que incorpora no sólo posibles mejoras en la eficacia y eficiencia de la acción pública, sino, además, la participación cívica por parte de la ciudadanía, reconciliando así los dos polos que vertebran buena parte de la discusión en torno a la crisis del Estado del bienestar (Offe, 1990).

De hecho, además de la participación cívica, dos han sido las funciones que han caracterizado a las asociaciones voluntarias, desde las clásicas aportaciones pluralistas, hasta las más recientes en torno a las reflexiones de la democracia asociativa; a saber: la mediación o public advocacy role y la provisión de servicios o service role $e^{3}$. La primera, centrada en la canalización, defensa y representación de intereses, o más en general, demandas de la ciudadanía o un grupo específico de ésta. La segunda, a través de la provisión de servicios, ya sea a miembros de la asociación, ya sea a personas que no pertenecen a ella.

Ahora bien, en el marco de las tendencias hacia la new governance, y en concreto, el pluralismo del bienestar, la segunda de las funciones enunciadas se ha visto ampliada por el hecho de que las asociaciones cumplen, así mismo, una función de complementación o suplementación de los servicios que provee el Estado del bienestar. Ya no se trata únicamente de que las asociaciones presten servicios, sino que prestan servicios en coordinación con las entidades públicas, o incluso proveen servicios públicos ${ }^{4}$. Se busca en su acción no sólo efectos sobre la dinámica democrática, en términos de capacitación cívica de sus miembros o de defensa y canalización de intereses, sino también de rendimiento o mejora en la provisión de servicios públicos, tal y como apunta la literatura sobre la democracia asociativa (Cohen y Rogers, 1995).

Ahora bien, aún delimitadas sus funciones, el fenómeno presenta un problema de demarcación, por cuanto, en general, aparece como aquello que no es ni estado, ni mercado, como una amalgama de organizaciones que, aparte de eso, pueden no tener ningún otro rasgo en común (Seibel y Anheier, 1990). Esta "definición residual» (Levitt, 1973) refleja, básicamente, un estiramiento conceptual que hace difícil su análisis, pero también la gestión del pluralismo del bienestar ${ }^{5}$. Ante ello, se plantea la necesidad de formular definiciones concep-

3. En este sentido, Salamon, Hems y Chinnock (2000) se refieren a las siguientes funciones o roles de las entidades voluntarias: personal or citizen advocacy (participación cívica), public advocacy (defensa de intereses), policy advocacy (participación en el diseño de políticas públicas) y service role (prestación de servicios).

4. El término suplementación, menos usual, se debe a Hirst (1993). Ahora bien, en la literatura sobre el tercer sector, suplementación y complementación se refieren a dos sub-modelos específicos para la provisión conjunta de servicios entre estado y asociaciones, además de aquéllos en los que domina o bien el estado, o bien las asociaciones (por ejemplo, Kramer, 1987).

5. El término estiramiento conceptual se debe a Sartori (1991). Este fenómeno se produce cuando una definición es tan genérica, es definida por tan pocos atributos, que deja de tener capacidad para definir con precisión un fenómeno. 
tuales más precisas. En general, siguiendo a Salamon y Anheiner (1992), se han dado cuatro tipos. A saber: legal, funcional, económica y estructural-operativa.

La primera toma como criterio la legislación de cada país, gobierno o agencia gubernamental. La funcional se refiere al ámbito de actuación de las entidades. La económica, al origen de los recursos económicos, que no deben provenir de la venta de bienes y servicios. La estructural-operativa, propuesta por Salamon y Anheiner (1992: 11), se refiere a cinco criterios. En concreto, señalan que las entidades han de ser organizaciones:

a) Formales, esto es, han de presentar cierto grado de institucionalización.

b) Privadas, es decir, «institucionalmente separadas del gobierno».

c) Sin ánimo de lucro: sus miembros no deben obtener beneficios procedentes de la venta de bienes y servicios en el mercado.

d) Autónomas, esto es, capaces de controlar sus propias actividades.

e) Voluntarias: la mayoría de sus miembros han de ser voluntarios, aunque no es preciso que todos lo sean.

Cada una de estas definiciones presenta ciertas ventajas e inconvenientes respecto a las otras, aunque la última pudiera considerarse la más oportuna. La legal, por ejemplo, parece económica y rigurosa, pues sólo utiliza un criterio como principio clasificatorio, pero es poco apta desde una perspectiva analítica, porque no permite hacer comparaciones (entre países, gobiernos o agencias gubernamentales) ${ }^{6}$. La funcional proporciona amplia información, pero enfrenta el problema de incompatibilidad entre categorizaciones de funciones y, por lo general, no es económica, pues los listados deben ser muy amplios en el intento de recoger todos los sectores o ámbitos en los que desarrollan su labor las asociaciones voluntarias. La económica es rigurosa, pero desatiende, por ejemplo, el carácter voluntario de los miembros o incluso la independencia respecto al Estado, factores clave de la naturaleza del tercer sector. La definición estructural-operativa, aunque implica recabar más información que en el caso de las otras definiciones, es rigurosa, permite comparaciones y atiende a aspectos básicos de la naturaleza que se le supone al fenómeno, por lo que suele considerarse la más oportuna.

Ahora bien, ¿qué definición manejan las agencias gubernamentales?, y sobre todo, ¿qué consecuencias tiene para la marcha del pluralismo del bienestar? A esto se dedica el próximo apartado.

\section{Pluralismo del bienestar como problema de información}

Básicamente, el pluralismo del bienestar implica la delegación de la prestación de algún servicio a una entidad del tercer sector por parte de una agencia gubernamental. ¿Hasta qué punto es eficiente esta nueva forma de provisión de ser-

6. Daría lugar a cierto parroquialismo, por usar el término opuesto al de estiramiento (Sartori, 1991). 
vicios públicos? Evaluarlo supondría saber si la agencia gubernamental conoce la acción que desarrolla la entidad elegida para la provisión. Cuestión que supone enfrentar el análisis de la relación entre tercer sector y agencias gubernamentales como un problema de información, tal y como propone el modelo o juego principal-agente, así como atender a la forma en que es diseñada y gestionada tal relación, para lo que puede ser útil atender al estilo político de interacción que subyace en ella.

\subsection{Información y costes de agencia: sobre la eficiencia de la relación entre tercer sector y agencias gubernamentales}

Más concretamente, la imagen del juego entre principal y agente puede resultar de interés, pues plantea un problema estratégico de carácter informativo similar al de las relaciones entre Estado y tercer sector. Por principal se entiende aquella persona o actor que encarga a un agente — otro actor - la realización de un servicio, ya sea porque no puede realizarlo por sí mismo, ya sea porque suponga una reducción de costes. Para ello establece un contrato, cuya eficiencia depende del conocimiento que el principal posea de la labor que realiza el agente.

Ahora bien, la relación entre principal y agente implica ciertos costes a los que suele denominarse costes de agencia. Éstos incluyen los que implican la búsqueda de información sobre los servicios, que está en condiciones de prestar el agente, los derivados del establecimiento del contrato, así como el control o la supervisión del cumplimiento de éste. Cabe suponer que si los costes, ya sea de información, contrato o control, sobrepasan los costes que supondría la provisión directa por parte del principal, el contrato será ineficiente ${ }^{7}$.

Así pues, lo que plantea este modelo principal-agente es, fundamentalmente, un problema de información: la que el principal posee sobre la naturaleza y acción del agente. En nuestro caso, el principal sería el Estado, pues mediante diversos ejercicios de facilitación financia (monetaria o no monetariamente) servicios que prestan las entidades sin fin de lucro. En este marco, cabe suponer que si el Estado desconoce la naturaleza o la acción que desarrollan las entidades, puede estar incurriendo en una provisión ineficiente, ya sea porque las entidades no desarrollen bien los servicios, ya sea porque para saberlo debería incurrir en altos costes de agencia, o sea, de supervisión del cumplimiento del «contrato».

De forma más concreta, en consideración a dos factores, como son la información que maneja el principal y la naturaleza de los segundos, cabe plantear diversos resultados de la interacción entre ambos en términos de los costes de agencia que acarrea la interacción. Por lo que se refiere al primer factor, pueden diferenciarse dos situaciones: información perfecta, aquélla en la que cada actor conoce las acciones que realizan otros actores, y la contraria, información imper-

7. Sobre el juego principal-agente puede consultarse Waterman y Meier (1998) o Gardner (1996). 
fecta o incertidumbre. En nuestro caso, si el Estado, como principal, conoce la acción de sus agentes - entidades - en todo momento, puede decidir si continuar o no con su relación o contrato sin incurrir en costes de agencia. En cambio, en una situación de incertidumbre deberá correr con ciertos costes de agencia para asegurar la eficiencia del contrato.

La naturaleza de los agentes se refiere al grado en que éstos son o no homogéneos entre sí en cuanto a su tamaño, recursos, etc. o, más en general, en cuanto a los límites relacionados con su naturaleza y capacidad de proveer servicios. Si las entidades — agentes - son heterogéneas entre sí y el principal no tiene conocimiento sobre ello, debe establecer el mismo tipo de contrato con todos los agentes, perdiendo así parte de la información e incurriendo, pues, en costes de agencia. $\mathrm{O}$ sea, no es lo mismo otorgar una subvención a una pequeña asociación local de discapacitados que a una cooperativa o a una entidad que cuenta con recursos y personal propios. Es preciso, pues, que las agencias gubernamentales tengan un conocimiento detallado de la naturaleza de las entidades con las que colaboran.

De hecho, en la literatura sobre este fenómeno, suele hablarse de que del mismo modo que existen «fallos del mercado» $\mathrm{y}$ "fallos del Estado» que justifican la presencia de las entidades voluntarias como agente de provisión de servicios, también cabe referirse a "fallos del tercer sector». Éstos se refieren, básicamente, a las siguientes cuestiones ${ }^{8}$ :

- Amateurismo: la baja capacidad profesional de las personas que desarrollan su labor en las entidades o, cuando menos, la baja capacidad de atracción de personal técnico dada su, en general, escasez de recursos.

- Provisores centrífugos: por el hecho de centrarse, por lo común, en sectores específicos de actuación, a diferencia de los servicios de carácter universalista del Estado del bienestar.

- Inexistencia de economías de escala: la escasez de recursos, su reducido tamaño, pero sobre todo el hecho de ser provisores centrífugos, limitan la necesaria generación de economías de escalas por parte de pequeñas entidades que procuren la provisión conjunta de servicios o el aumento de su capacidad de información y actuación.

Así pues, desde la perspectiva analítica aquí presentada, las agencias gubernamentales (como principal) deberían tener información sobre las entidades voluntarias (agentes) referida, al menos, a, por un lado, los cinco criterios de la definición operativo-estructural que dan cuenta de su naturaleza como entidades voluntarias y, por tanto, la medida en que las entidades son homogéneas o no entre sí, y, por otro lado, información sobre su capacidad de prestación de servicios, esto es, la relativa a la existencia o no de los fallos del tercer sector recién mencionados. 
Tabla 1. Requisitos informativos del pluralismo del bienestar.

\begin{tabular}{llll}
\hline \multicolumn{3}{c}{ Valores } \\
\hline Requisitos & Información & Perfecta (completa) & Imperfecta (incertidumbre) \\
& Homogeneidad agentes & Sí & No \\
\hline Costes de agencia & - & + \\
\hline Solución (situación tipo) & Óptimo social & Subóptimo social \\
\hline
\end{tabular}

De lo hasta aquí expuesto, se deriva la existencia de dos situaciones tipo. Una de óptimo social, en donde los costes de agencia serían mínimos, producto de la conjunción de la existencia de información perfecta por parte del principal —el Estado_ - y homogeneidad de los agentes — las entidades_Otra, de subóptimo social, derivada de la presencia de altos costes de agencia en razón del desconocimiento o la incertidumbre del principal respecto a la acción y capacidad de los agentes, así como por el hecho de presentar éstos una muy heterogénea naturaleza (tabla 1 ).

En resumen, el marco analítico consideraría dos variables, a saber: la situación de información que poseen las agencias gubernamentales respecto a las acciones y a la capacidad de las entidades con respecto a la provisión de bienestar, y el conocimiento de la mayor o menor homogeneidad de la naturaleza de las entidades con las que interactúan. Cuanto más cerca de la situación de información perfecta y mayor la homogeneidad, menores los costes de supervisión o agencia; y, con ello, el pluralismo de bienestar, en la forma de relaciones entre Estado y tercer sector, se encontrará más cerca de la situación de óptimo social. Al contrario, cuanto mayor la incertidumbre y la heterogeneidad de las entidades, mayores los costes de agencia y, por tanto, más cerca de la situación de subóptimo social se encontrará el pluralismo del bienestar, tal y como se presenta en el gráfico 1 .

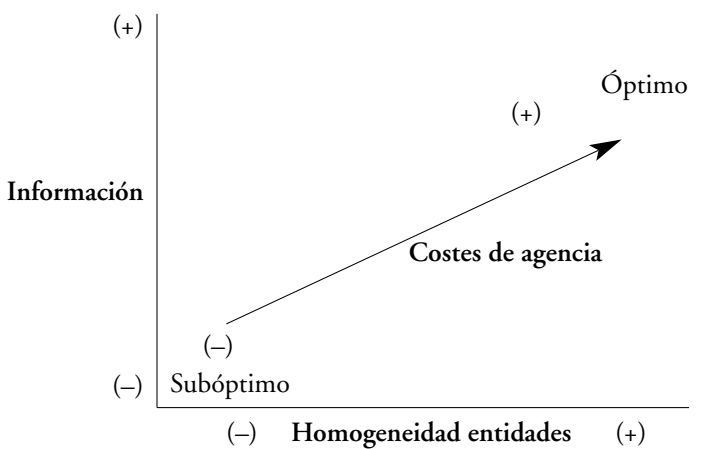

Gráfico 1. Marco analítico para la evaluación de la calidad del pluralismo de bienestar: calidad informativa de la relación entre agencias gubernamentales y tercer sector. 


\subsection{Los estilos politicos de interacción: la gestión de la información y el papel asignado a las entidades sin ánimo de lucro}

Evidentemente, además de la cantidad y calidad de la información de las agencias gubernamentales sobre las entidades sin ánimo de lucro, cabe considerar como un aspecto relevante de sus procesos de interacción el modo en que esta información es gestionada. Esto es, aspectos tales como la existencia o no de marco legal que regule la naturaleza de las entidades o los requisitos que deben cumplir para establecer procesos de interacción con las agencias gubernamentales, para actuar como agentes respecto a ellas.

En este sentido, se pudieran definir diversos estilos políticos, entendidos como formas modales de interacción entre entidades y agencias gubernamentales ${ }^{9}$. Para ello, cabe atender fundamentalmente a dos aspectos. Por un lado, la definición o conceptualización que las agencias gubernamentales realizan de las entidades, la forma en que son percibidas, las tareas que se cree deben realizar, así como los recursos que deben intercambiar con el Estado. A grandes rasgos, pudiera considerase que tales conceptualizaciones se sitúan en un continuum desde la consideración de las entidades como mecanismos de transmisión de demanda política, a la consideración de éstas como agentes de provisión de bienestar; o lo que es lo mismo, las funciones de public advocacy y service role, tal y como las delimitan Salamon, Hems y Chinnock (2000), a las que se ha hecho referencia más arriba.

Por otro lado, cabe considerar el marco institucional por el que se regula la interacción, al entender que éste refleja percepciones acerca de la naturaleza y tareas que debieran desarrollar las entidades como agentes respecto al Estado. Más concretamente, a través de la normativa vigente o las declaraciones de informantes cualificados podría conocerse la naturaleza de las entidades, los mecanismos establecidos para su inclusión en los registros, la gestión, el seguimiento y la actualización de éstos, así como la regulación al acceso a ayudas públicas de diversa índole.

Resulta evidente que el estilo político viene conformado por otros factores de carácter menos formalizado que igualmente pueden configurar la interacción entre agencias y entidades y, por tanto, informar de la naturaleza de éstas últimas - relaciones informales, superposición de los miembros, etc.(Navarro, 1999). Aunque se trata de aspectos relevantes, permitirían conocer la dinámica y las pautas de interacción que realmente mantienen asociaciones y agencias gubernamentales, asunto ajeno a los objetivos de nuestra investigación. Desde nuestro punto de vista, la definición de las entidades por parte de las agencias gubernamentales y el marco institucional que regula su interacción aparecen aspectos significativos y suficientes para delimitar el estilo político que caracteriza su interacción y, más concretamente, para conocer aspec-

9. Siguiendo a Richardson (1982), por estilos políticos se entienden las configuraciones institucionales que delimitan la forma en que autoridades públicas y actores civiles interactúan para resolver problemas. 
tos básicos de la calidad y cantidad de información sobre la que se basa la relación principal-agente que establecen agencias gubernamentales y entidades.

\section{El análisis de los registros de entidades de las agencias gubernamentales}

Para aplicar empíricamente el marco analítico expuesto en los apartados anteriores, se ha desarrollado un estudio de caso tomando como ejemplo Andalucía. Como tal, este diseño de investigación sólo trata de probar la utilidad del marco analítico propuesto, así como desarrollar algún instrumental de análisis empírico, sin pretender con ello su validez como modelo explicativo del conjunto de relaciones que mantienen tercer sector y agencias gubernamentales en Andalucía, ni tampoco que sus resultados sean exportables a otros casos. Se trata, pues, de un estudio de caso orientado a la generación de hipótesis por el que se pretende avanzar explicaciones que deberán mejorarse analíticamente y cotejarse con más casos (Bartolini, 1986).

Para conocer la cantidad y la calidad de la información que las agencias gubernamentales poseen sobre las entidades en Andalucía, el estudio trató de recabar los registros de entidades que aquéllas poseen y, en el caso de que existiesen, analizar posteriormente su contenido. El ejercicio se centra en tres políticas básicas del Estado del bienestar, como son salud, educación y bienestar social, además de las de fomento de la igualdad entre mujeres y hombres. Así mismo, se consideraron las agencias de ámbito autonómico y local, además del Registro General de Entidades, en donde deben estar incluidas todas las entidades existentes en Andalucía. En concreto, para cada agencia se estudió la denominación o definición de las entidades, la existencia o no de marco legal, el papel asignado a cada una de ellas a partir de lo anterior, así como la información requerida por las agencias a las entidades y la realmente contenida en los registros. Además, se entrevistó a algún técnico que tuviese como tarea la gestión del registro para que, como informantes cualificados, nos informasen sobre aspectos referidos a los estilos políticos de interacción (actualización de los registros, información que se pide realmente a las entidades, qué concepción se tiene de éstas, etc.) ${ }^{10}$.

\subsection{Denominación, marco legal y soporte de los registros: mosaico desigual y fluidez conceptual}

En la tabla 2 se presentan los registros o bases de datos que hemos analizado para nuestra indagación. En ella se ha reflejado la denominación que recibe el registro, si existe un marco legal específico que lo regula, así como el soporte en el que ha sido posible recopilar la información.

En la gran mayoría de los casos, las agencias poseen dos tipos de registros: un registro de entidades y un listado de entidades que han pedido y/o que han

10. Para más detalles del diseño metodológico de la investigación se remite al capítulo cuarto de Navarro (2001), en donde se da cuenta de las agencias visitadas y la información recabada, así como de las entrevistas realizadas. 
Tabla 2. Registros de entidades: denominación, marco legal y soporte.

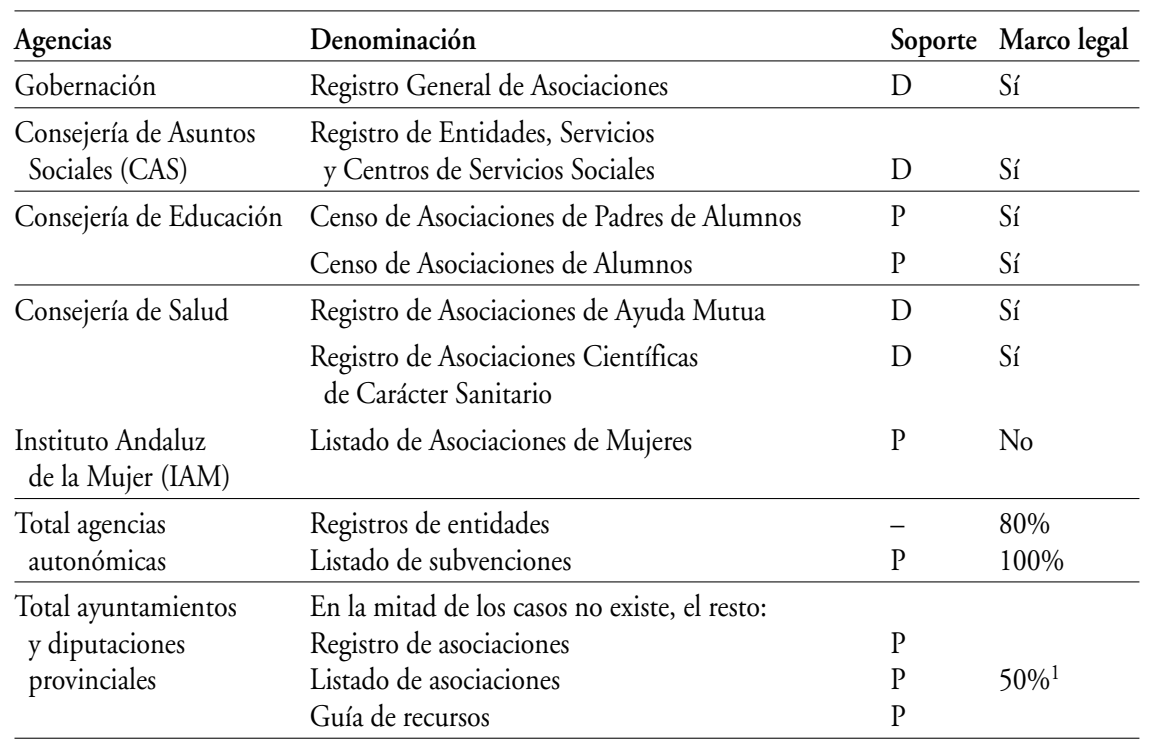

1. Decreto de alcaldía o incluido en el Reglamento de Participación Ciudadana.

recibido subvenciones. Por lo común, el primero viene a funcionar como una especie de cuasi censo, en el que se registran las asociaciones que trabajan en el ámbito de actuación de la agencia correspondiente, o bien todo tipo de asociaciones cuando se trata del Registro General de Entidades y, sobre todo, el departamento de participación ciudadana a nivel municipal ${ }^{11}$. Para este tipo de registros suele existir un marco legal específico en el caso de las consejerías, y para algunas entidades de gobierno municipal, ya sea en el marco de los reglamentos de participación ciudadana en los que se incluye el registro, ya sea por la existencia de un marco normativo específico para el registro, como ocurre en el caso del ayuntamiento de Málaga.

En general, la existencia de registros y la información recogida en ellos es muy desigual. Existen registros en las agencias gubernamentales de carácter autonómico (aunque no siempre en soporte magnético ni con programa específico de gestión de datos), pero no en gran parte de los gobiernos locales. Además, en estos casos, de no existir un marco normativo específico — en forma de reglamento de participación o para el registro propiamente dicho-, la existencia de listados depende o bien de la labor de un departamento que se encarga de hacer una guía de recursos, en la que se incluyen las asociaciones junto a

11. En este caso, cabe incluir a la Diputación de Córdoba, pues existe un registro de asociaciones a cargo del Consejo Provincial de Participación Ciudadana. 
organismos públicos y privados, o bien la labor de personas que creen necesaria la recopilación de esta información para el desempeño de su trabajo en departamentos específicos ${ }^{12}$. En su conjunto, se trata de un agregado descoordinado de información que nos presenta el mundo de las asociaciones o el tercer sector en la forma de un mosaico muy desigual en cuanto a la información existente y a la forma en que es gestionada.

\subsection{La definición de entidad: la "fluidez conceptual» y el papel asignado a las entidades}

Además del grado de fragmentación y descoordinación que presentan los registros, una de las conclusiones más relevantes del trabajo desarrollado se encuentra en la "fluidez conceptual» que presentan las entidades sin ánimo de lucro entre las agencias gubernamentales consultadas. En primer lugar, porque no suelen ofrecer una definición clara y explícita de las entidades, más allá de lo establecido a través de diversas normas o legislaciones, debiendo deducirse de caracterizaciones generales que aparecen en diversos documentos. En segundo lugar, y como consecuencia de lo anterior, porque no existen mínimos comunes por los que llegar a una definición válida para todas las agencias consultadas.

En todos los casos las entidades son conceptualizadas mediante definiciones de tipo legal, ya sea a partir de la legislación general existente al efecto (Ley $191 / 1964)$, ya sea a partir de marcos normativos específicos delimitados por cada agencia gubernamental. En el caso de agencias gubernamentales que poseen un ámbito de actuación más amplio, con competencias sobre diferentes asuntos o sectores de población, suele hacerse uso, asimismo, de una definición funcional, diferenciando tipos de entidades según diversos criterios: fórmula jurídica, sector o sectores de actividad, población objetivo, tipos de socios, etc. Como se indicó en el primer apartado, esta definición da lugar a amplios y desiguales listados de tipos que dificultan la comparación entre registros, y no son rigurosas, pues los fines de las entidades pueden ser múltiples y cambiantes. En suma, en cuanto a la definición de las entidades, existe un fuerte parroquialismo departamental, esto es, cada departamento maneja una definición específica y diferenciada de las entidades con las que interactúa.

En ningún caso las definiciones encontradas se acercan a la delimitación "estructural-operativa». En todo caso, se parte del supuesto de que son organizaciones formales, pues deben entregar unos estatutos que así lo garantizan; asimismo, este documento presupone que son privadas (sin control de organismos públicos) y sin fin de lucro; aunque en ningún caso puedan garantizarse tales hechos. Los propios entrevistados declaran tener dudas acerca de la naturaleza de las entidades y, en concreto, acerca de su carácter no lucrativo,

12. De hecho, durante las entrevistas realizadas se nos han ofrecido diversos listados elaborados por personas que trabajan en las agencias gubernamentales o alguna plataforma o federación de asociaciones, aunque en todo caso son de tipo parcial y reflejan meramente datos identificativos de las entidades, por lo que se ha preferido no incluirlos en el análisis. 
por lo que recomiendan que los datos sean «cogidos con pinzas». Esto es, la información que recogen los registros no permite aplicar cinco criterios que dan cuenta de la definición estructural-operativa, en todo caso, se dan por supuestos. Ello constituye un primer indicador del grado en que las entidades son tratadas de forma homogénea en cada registro aunque no lo sean, o cuando menos, puedan no serlo, que a los efectos del marco analítico propuesto tiene el mismo efecto, esto es, se trata de desinformación.

Aun así, del análisis de contenido de la documentación consultada cabe extraer tres modelos de la concepción que la administración posee acerca de las entidades con las que interactúa y, más concretamente, de la labor que deberían realizar:

1) Modelo «canalización de demanda»: se utiliza preferentemente la definición legal, en la que, en atención a los contenidos de ésta, se asigna la función de canalización de la demanda y representación de intereses. Las entidades aparecen principalmente como un ámbito de participación, defensa y representación de intereses o, si se prefiere, la función de public advocacy. Éste es el caso del Registro General de Asociaciones (RGA), así como en parte de los gobiernos locales, los cuales sólo exigen la presentación del número del RGA y los estatutos.

2) Modelo «intermedio»: se utiliza la definición legal, pero ésta incluye, además de la canalización de la demanda, el que las entidades realicen ciertas actividades que toman como beneficiarios, fundamentalmente, a sus miembros. Este modelo está representado por las consejerías de Salud y Educación, así como el IAM y algunos gobiernos municipales. Por lo que se refiere a la Consejería de Educación, queda claramente especificado que las asociaciones de padres de alumnos y las asociaciones de alumnos son entidades sin fin de lucro que deben tanto participar en el "gobierno» de los centros educativos en los términos que establece la ley — representando a padres y alumnos, respectivamente, en los consejos escolares-, como desarrollar actividades que consideren interesantes para los centros, públicos o privados - principalmente culturales y deportivas- . Se trata de entidades que actúan sobre un sector específico y en un ámbito concreto, pues de hecho no puede existir más de una asociación por centro escolar, sea público o privado. Por último, entre los niveles de gobierno local, parece estar dándose cada vez con mayor intensidad una orientación hacia la prestación de servicios, sin obviar o abandonar la tradicional canalización de la demanda política (Navarro, 1998). Esto es, además de la función de public advocacy, se enuncia la de service role, aunque fundamentalmente se trate de servicios o actividades propias sin que se den procesos de complementación o suplementación con respecto al Estado.

3) Modelo "prestación de servicios»: en este caso, además de la definición legal, se insiste, sobre todo, en la prestación de servicios, en la función de service role. Éste sería representado por los registros de subvenciones, pues éstas se conceden con la finalidad de prestar un servicio o realizar una actividad. 
Pero, sobre todo, es el caso del Registro de Entidades y Centros de Servicios Sociales de la CAS. De hecho, la definición engloba tanto entidades con fin de lucro, como entidades sin fin de lucro, pues el criterio básico de definición es la prestación de un servicio. En concreto, se señala que es «Entidad de Servicios Sociales toda persona física o jurídica de cualquier clase que actúe en los sectores de Servicios Sociales, que se proponga, con voluntad de permanencia, la asunción de la titularidad de un Servicio o Centro» (Orden 29 de febrero de 1996). La diferenciación sólo queda especificada mediante la declaración que cada entidad haga en el momento de pedir su incorporación al registro, información que, como veremos más adelante, no queda recogida a través de la convocatoria de subvenciones, aunque a ellas pueden concurrir tanto entidades como personas físicas.

Se trata, pues, de un conjunto amplio pero heterogéneo de entidades que, en principio, es conceptualizado y tratado de forma similar por las agencias a través de la definición legal y funcional. Pautas de interacción que dificultan adecuar sus interacciones al óptimo social que pudiera caracterizar a las relaciones entre el principal (gobierno) y las agencias (entidades) en el desarrollo del pluralismo del bienestar. Aunque, en todo caso, puedan establecerse diferencias en atención a la importancia que se le conceda a la provisión de servicios (service role) frente a la de participación y defensa de intereses (public advocacy).

Ahora bien, si estas son las definiciones que manejan las agencias gubernamentales, que presuponen la demanda de determinada información a las entidades, ¿qué información se les reclama a las entidades para ser incluidas en los registros? y, además, ¿qué información poseen realmente los registros?

\subsection{Información contenida en los registros}

El análisis de la información contenida en los registros muestra la desinformación que poseen las agencias con respecto a las entidades con las que interactúan, más allá de los datos básicos referidos a nombre y domicilio de cada asociación, lo que se pudiera denominar «información censal mínima».

Para conocer la cantidad y calidad informativa de los registros, se ha diferenciado entre «información normativa» (IN), aquélla que debe quedar recogida en los registros según las normas que regulan su gestión y mantenimiento, e «información real» (IR), la que realmente existe en las bases de datos analizadas. Cada una de estas informaciones - o campos- quedan especificadas en la tabla 3. Para su correcta interpretación, se debe tener en cuenta que la presencia de un asterisco indica que se trata de información normativa que debería constar, mientras que el dato que se muestra da cuenta, primero, que se trata de «información real» $y$, segundo, indica el porcentaje de asociaciones o entidades integradas en los registros que poseen la información de que se trate.

En este punto, debe considerarse que el análisis se ha limitado a los registros recopilados en soporte magnético; aunque no se haya obviado la infor- 
Tabla 3. Información contenida en los registros: indicadores de calidad informativa y coincidencia con el Registro General de Asociaciones.

\begin{tabular}{|c|c|c|c|c|c|c|c|c|c|}
\hline \multirow[b]{2}{*}{ Rasgos } & \multicolumn{9}{|c|}{ Bases de datos } \\
\hline & $R G A$ & CAS & $S A L U D$ & $I A M$ & EDUCACIÓN & $L O C A L$ & $S U B$ & $I C$ & \\
\hline Número de registros (entidades) & 26.453 & 3.195 & 417 & 1.522 & 604 & 3.000 & - & $n$ & $\%$ \\
\hline \multicolumn{10}{|l|}{ I. Identificación de la entidad } \\
\hline 1. No identificación Gobernación & 100 & & 4 & $*$ & & & & 0 & 0 \\
\hline 2. No identificación propio & & 100 & * & & 100 & * & & & \\
\hline 3. Nombre & 100 & 100 & 100 & 100 & 100 & 100 & 100 & 6 & 100 \\
\hline 4. Domicilio & 94 & 100 & 100 & 100 & 100 & 100 & 100 & 6 & 100 \\
\hline 5. Distrito/código postal & & 92 & 98 & 99 & 100 & 100 & 100 & 5 & 83 \\
\hline 6. Municipio & 94 & 100 & 100 & 100 & 100 & 100 & 100 & 6 & 100 \\
\hline 7. Provincia & & 100 & 100 & 100 & 100 & 100 & * & 5 & 83 \\
\hline 8. Teléfono & & 74 & 93 & * & 100 & * & & & \\
\hline 9. $\mathrm{Fax}$ & & * & 19 & & * & & & & \\
\hline 10. Número patronal & & $*$ & & & & & & & \\
\hline 11. CIF & & * & 63 & $*$ & & & 100 & & \\
\hline 12. Fecha/año constitución & & * & & & & & & & \\
\hline 13. Nombre representante legal & 100 & $*$ & & $*$ & $*$ & $*$ & 100 & 1 & 17 \\
\hline \multicolumn{10}{|c|}{ II. Criterios sobre la naturaleza de la entidad: características de la entidad } \\
\hline 14. Tipo (persona jurídica) & & $*$ & $*$ & & & & & & \\
\hline 15. Ámbito geográfico & 93 & * & 100 & * & & & & 1 & 17 \\
\hline 16. Tipo: sector principal atención & 95 & $*$ & 100 & * & & 100 & & 2 & 33 \\
\hline 17. Otros sectores atención & & $*$ & & & & & & & \\
\hline 18. Gestiona centro/servicios & & * & & & & & & & \\
\hline
\end{tabular}

III. Capacidad de la provisión de servicios: datos sobre recursos

19. Personal

20. Tipos de personal

21. Gastos anuales

22. Procedencia financiación

IV. Capacidad de la provisión de servicios: datos relaciones con agencias gubernamentales

23. Acreditación

24. Gestiona centros/servicios públicos

25. Convenios/conciertos

26. Ha recibido subvención

V. Datos específicos relativos a solicitud/concesión de ayuda (subvención) no incluidos en los anteriores

27. Programa anual de actividades

28. Sector pobl. Objetivo/modalidad

29. Descripción programa/actividad

30. Cantidad solicitada

31. Cantidad concedida 
Tabla 3. Información contenida en los registros: indicadores de calidad informativa y coincidencia con el Registro General de Asociaciones (continuación).

\begin{tabular}{|c|c|c|c|c|c|c|c|}
\hline \multirow[t]{2}{*}{ Rasgos } & \multicolumn{7}{|c|}{ Bases de datos } \\
\hline & $R G A$ & $C A S$ & SALUD & $I A M$ & EDUCACIÓN & LOCAL & $S U B \quad I C$ \\
\hline \multicolumn{8}{|l|}{ Indicadores } \\
\hline Total de I a IV («normativos») & 7 & 21 & 13 & 14 & 9 & 9 & 7 \\
\hline Total V («normativos») & 0 & 0 & 0 & 0 & 0 & 0 & 5 \\
\hline Total «normativos» & 7 & 21 & 13 & 14 & 9 & 9 & 12 \\
\hline Total «reales» & 7 & 7 & 11 & 5 & 7 & 6 & 7 \\
\hline Diferencia R-N & 0 & -14 & -2 & -9 & -2 & -3 & -5 \\
\hline $\mathrm{ICI}=(\mathrm{R} / \mathrm{N})^{*} 100$ & 100 & 33 & 85 & 36 & 78 & 67 & 58 \\
\hline Posee datos censales mínimos & Sí & Sí & Sí & Sí & Sí & Sí & Sí \\
\hline
\end{tabular}

IC: Índice de coincidencia con RGA.

ICI: Índice de calidad informativa.

RGA: Registro General de Asociaciones, Consejería de Gobernación; CAS: Consejería de Asuntos Sociales; Salud: Consejería de Salud; IAM: Instituto Andaluz de la Mujer, Educación: Consejería de Educación y Ciencia; Local: agencias locales (ayuntamientos y diputaciones), Sub: listados de subvenciones.

mación recogida en papel. Por otra parte, que se ha integrado la información referida a los niveles de gobierno local, por un lado, y a las diversas convocatorias y listados de subvenciones, por otro, ya que internamente a tales grupos los registros requieren y ofrecen información similar.

Del análisis de la tabla 3 puede concluirse, en primer lugar, que la información normativa no se refiere, por lo común, ni a los cinco criterios que permiten conocer la naturaleza de las entidades, en atención a la definición estructural-operativa, ni tampoco aquéllos otros relacionados con su capacidad o «fallos» como agentes provisores de bienestar. Esto es, de partida, la información que reclaman los registros configura una «situación normativa» más cerca de la de subóptimo que de la de óptimo, según el marco analítico propuesto. Si bien existen diferencias, pues la información normativa viene a reflejar la definición y las funciones asignadas comentadas en el apartado anterior.

De hecho, pudiera señalarse la existencia de dos grandes modelos a este respecto. Por un lado, el «modelo censo», que se limita a requerir a las entidades información mínima de tipo censal, por otro lado, el «modelo prestación de servicios», que les exige, además, información sobre los recursos de que disponen las entidades, así como las actividades que desarrollan, en unos casos, como la Consejería de Asuntos Sociales (CAS), sobre sus actividades y sectores de actuación en general, en otros, como en el caso de las convocatorias específicas de subvenciones, sobre la actividad para la que piden la ayuda económica.

En segundo lugar, el análisis de la información real abunda en el grado de incertidumbre en el que se desarrollan la interacción entre agencias gubernamentales y entidades voluntarias. En este sentido, y con la finalidad de obte- 
ner una visión global del contenido informativo de los registros, hemos construido dos indicadores sintéticos:

- Indicador de Calidad Informativa (ICI): porcentaje de información que existe en cada registro - base de datos - con relación al total de información que debería ofrecer según cada marco normativo específico. Esto es, $\mathrm{ICI}=(\mathrm{IR} / \mathrm{IN})^{*} 100$. Siendo su recorrido igual al intervalo $[0,100]$, cuanto mayor es el índice, mayor es el grado de adecuación entre información normativa e información real.

- Indicador de Coincidencia con el Registro General de Asociaciones (IC): número de registros — bases de datos - que ofrecen una información similar a la que ofrece el Registro General de Asociaciones de la Consejería de Gobernación. Esto es, IC = (bases que poseen información similar al RGA/total bases consideradas)* 100 . Con un recorrido igual al intervalo $[0,100]$, este indicador debe interpretarse en sentido creciente, cuanto mayor sea, mayor será el grado de coincidencia entre la información que contiene el RGA y las otras bases de datos analizadas.

Respecto al ICI, cabe destacarse su diversidad. En general, y a excepción del Registro General de Asociaciones, el indicador se sitúa en torno al 60\%, siendo la media del 65,28\%. Por encima de ésta se sitúan el registro de las Asociaciones de Ayuda Mutua de Salud y el registro del Instituto de la Mujer, con índices en torno al 80\%. Por debajo, el registro de la CAS (33\%) y los listados o registros de subvenciones (58\%). El caso de Asuntos Sociales pudiera explicarse porque, paradójicamente, la Ficha de Entidades y Centros de Servicios Sociales es la que requiere un mayor volumen de información a las entidades, aunque posteriormente no sea registrada; mientras que los otros casos sólo requieren una información mínima, que sí es registrada.

En su conjunto, de lo anterior pudiera derivarse que la calidad informati$v a$ de los registros es de nivel medio, pero es destacable, sobre todo, que los datos recogidos en los registros se refieran básicamente a lo que podríamos denominar como "datos censales mínimos», esto es: nombre de la entidad, domicilio, código postal, municipio y provincia. De hecho, la adecuación entre «información normativa» e «información real» en el caso del Registro General de Asociaciones o en el del IAM, se explica porque estos registros requieren exclusivamente este tipo de información básica, que, por lo común, está presente en todas las bases de datos analizadas.

Respecto al Índice de Coincidencia, se evidencia cierta descoordinación con respecto al Registro General de Entidades. A pesar de que la información existente en la práctica totalidad de los registros analizados coincide con la información requerida en el Registro General de Entidades y, en concreto, la referida a la «información censal», es destacable que en ningún caso se recoja el número de identificación que asigna Gobernación en los otros registros.

En suma, del análisis del contenido informativo de los registros pudiera confirmarse, por un lado, que la propia información normativa que reclaman las agencias gubernamentales mediante sus respectivos registros dan cuenta de una 
situación de incertidumbre, tanto por lo que se refiere a la naturaleza de las entidades, como a su capacidad de prestación de servicios. Aunque, grosso modo, existan dos tendencias o modelos en cuanto a la relación que las agencias gubernamentales pretenden establecer con las entidades sin fin de lucro, desde la mera constancia censal, cuyo modelo prototípico es el Registro General de Asociaciones (Consejería de Gobernación), hasta la recogida de información orientada a la incorporación de las entidades a labores de producción y prestación de servicios, siendo el modelo típico el Registro de Entidades y Centros de Servicios Sociales (Consejería de Asuntos Sociales). Por otro lado, cabe concluir que, en general, el nivel de información es medio-bajo, pues, además de lo anterior, la información real es aún menor que la normativa, lo que abunda en el grado de incertidumbre existente en la relación entre principal y agente.

\section{A modo de conclusiones: estilos políticos de interacción y calidad del pluralismo del bienestar}

Los resultados del análisis expuesto en el apartado anterior, permiten caracterizar el estilo político que pretende desarrollar cada agencia gubernamental con respecto a las entidades del tercer sector y, por tanto, los rasgos básicos del diseño institucional del pluralismo del bienestar. En concreto, por lo que se refiere a la «información normativa», del análisis conjunto de las definiciones de las entidades y del contenido de los registros puede concluirse la existencia de dos modelos o estilos políticos. A saber:

- Estilo registral: se caracterizaría por el uso de una definición en el que se acentúa el carácter de canalización de demanda y participación política sobre la provisión de servicios, basado principalmente en una definición legal de las entidades y, en todo caso, en una definición funcional. Ello se refleja también en un modelo de gestión más cercano al tipo «censo» que al tipo "gestión de actividades», limitando su «información normativa» a la que hemos denominado «información censal mínima». El caso paradigmático sería el Registro General de Asociaciones, así como las diputaciones y los ayuntamientos, aunque también cabría incluir al IAM y las consejerías de Educación y Salud.

- Estilo gerencial: la definición de las entidades, aún basándose únicamente en criterios legales y funcionales, insisten en mayor medida que el caso anterior en la realización de prestación de servicios, ya sean servicios de titularidad pública, como en el caso de la CAS, o servicios que producen y proveen directamente las entidades a través de las actividades que les son subvencionadas. El modelo de gestión y la «información normativa» de sus registros se acercan en mayor medida al modelo de "prestación de servicios», en el que se requiere información acerca de la dinámica de las entidades, más allá de la información censal mínima.

Resulta evidente que el estilo político gerencial es el que se acerca en mayor medida al conjunto de requisitos informativos necesarios para el desarrollo del 


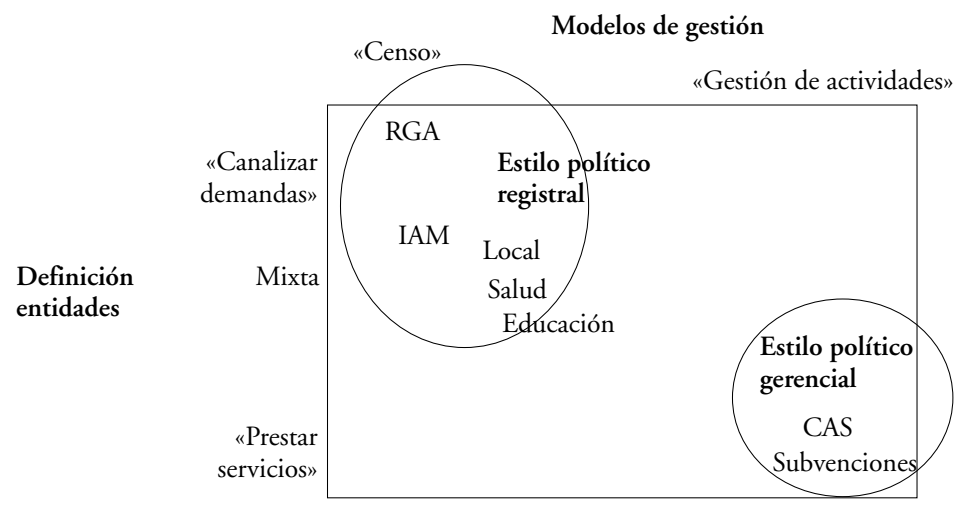

Gráfico 2. Los estilos políticos en la interacción entre agencias gubernamentales y entidades, según «información normativa».

pluralismo del bienestar, tal y como han sido definidos más arriba; tanto lo que se refiere a los criterios que dan cuenta de la naturaleza de las entidades (presentados en el apartado 1), como a su capacidad en tanto que agentes provisores de servicios (apartado 2). Ahora bien, a pesar de este hecho, el análisis empírico del desarrollo de los modelos, a través de la «información real» contenida en los registros, ha venido a mostrar que en la realidad todas las agencias se relacionan con las entidades del tercer sector mediante los patrones del modelo registral, lo que dificulta la creación de situaciones de óptimo social en la extensión del pluralismo del bienestar (gráfico 2).

De forma más específica, el análisis ha permitido detectar los siguientes problemas:

1) Fluidez conceptual: las definiciones que viene utilizando la Administración pública remiten únicamente a criterios legales y funcionales que, aunque rigurosos, no permiten discernir realmente el tipo de entidad que contienen sus registros.

2) Gestión de información: se manifiesta tanto en sentido vertical como horizontal. Por un lado, descoordinación vertical, pues, en general, no se asegura la necesaria coordinación entre el Registro General de Asociaciones, y, por otro, descoordinación horizontal, porque no suelen existir mecanismos de coordinación entre diferentes agencias gubernamentales en un mismo nivel de gobierno, por lo que se produce una situación de minifundismo departamental que dificulta el conocimiento y la supervisión de las relaciones que establecen entidades y gobiernos en su conjunto.

3) Incertidumbre: la información que sobre las entidades tienen las agencias es escasa, salvo la referida a su nombre y domicilio, particularmente la refe- 
rida a su naturaleza y, sobre todo, la relacionada con la actividad que realizan.

Estas deficiencias vienen a mostrar que el desarrollo del pluralismo de bienestar a través de la potenciación del tercer sector presentará, previsiblemente, una situación de subóptimo social, pues, por un lado, la fluidez conceptual provoca que en los registros aparezca un conjunto heterogéneo de entidades, de tal forma que las agencias deben crear convenios generales comunes a todas ellas, lo que provoca un aumento considerable de los costes de agencia; por otro lado, la descoordinación refuerza la situación de heterogeneidad antes descrita, pero además reduce la capacidad de supervisión de las agencias sobre las entidades, al no poder controlar las primeras los recursos que éstas reciben, incluso, de diversas agencias gubernamentales al mismo tiempo. Ello provoca el aumento de los costes de agencia y, aún más, pudiera producir redundancia y duplicación de actividades y apoyo a las entidades. Por último, la desinformación acerca de las entidades, tanto en cuanto a sus rasgos, como con respecto a sus actividades, recursos, personal, etc., crea una situación de creciente incertidumbre en las relaciones entre principal (agencias gubernamentales) y agentes (entidades), lo que dificulta el conocimiento de la adecuación de los segundos para la producción y distribución de servicios que propone el primero, pero también crea dificultades para establecer mecanismos de control y evaluación del primero sobre los segundos.

No obstante estas conclusiones, ha de considerarse que el presente artículo ha tratado de avanzar, de una forma básica, propuestas analíticas e instrumental metodológico para analizar y, si se quiere, evaluar el desarrollo del pluralismo del bienestar entendido como una relación principal-agente entre agencias gubernamentales y entidades del tercer sector. Los resultados muestran, para un caso concreto, los problemas a los que se puede enfrentar este nuevo modelo de provisión de servicios públicos.

Aunque el ejercicio analítico se ha realizado para un caso, cabe esperar que la situación sea similar en otros, pues en general el conocimiento y la información sobre las entidades del tercer sector es deficiente, no sólo para las agencias gubernamentales, sino también en el ámbito académico (Rodríguez Cabrero y Codorniú, 1996). En general, se trata de un problema que afecta al estudio de cualquier aspecto relacionado con este tipo de entidades, sobre las que, a pesar de su creciente importancia, existe aún poca reflexión conceptual, teórica y metodológica que permita ir desarrollando procesos de análisis y evaluación rigurosos. Sin duda, esto supone un límite importante del análisis realizado y sus posibilidades de aplicación a otros casos, aunque no cabe pensar que suponga erosión alguna del marco analítico en sí mismo.

No obstante, a este respecto debe destacarse que este artículo ha desatendido otro de los aspectos importantes de las entidades que conforman el tercer sector, como es su papel de actores políticos que procuran la articulación de intereses, así como la canalización y defensa de demandas de la ciudadanía. No 
cabe duda que la evaluación del pluralismo del bienestar debería considerar también esta dimensión, no ya desde la perspectiva de la eficacia en la provisión de servicios, sino más bien en atención a la mejora que ello introduce en la calidad democrática de nuestros sistemas políticos. Asunto éste que, conscientemente, no ha sido objeto de este artículo.

\section{Bibliografía}

AgUiAR, F. y otros (2001). Las entidades sin fin de lucro en Andalucía: proyecto de operación censal. Sevilla: IEA.

BARTOLINI, S. (1986). «Metodología de la investigación política». En BARTOLINI, S. y otros (eds.). Manual de Ciencia Política. Madrid: Alianza, p. 39-78.

Clarke, S. (ed.) (1998). Local autonomy and innovation. Londres: Sage.

COHEN, J.; Rogers, J. (1995). «Secondary Associations and Democratic Governance».

En Wrigth, E.O. (ed.). Associations and Democracy. Londres: Verso, p. 7-100.

Douglas, J. (1987). «Political Theories of Nonprofit Organization». En PowelL, E.

(ed.). The Nonprofit Sector: A Research Handbook. New Haven: Yale University Press, p. 43-54.

Gardner, R. (1996). Teoría de juegos. Barcelona: Antoni Bosch Editor.

HirTs, P. (1994). Associative democracy. Londres: Polity Press.

Hodgkinson, V.A.; Sumariwalla, R.D. (1992). «The Nonprofit Sector and the New

Global Community: Issues and Challenges». En MCCARTHY, K.D. y otros. The

Nonprofit Sector in Global Community. Washington: The Independent Sector, p. 485-508.

KoOIMAN, J. (ed.). Modern Governance. Londres: Sage.

KRAMER, R. (1987). "Voluntaries agencies and the personal social services». En Powell, W. (ed.). The Nonprofit Sector. A Research Handbook. New Haven: Yale University Press, p. 240-257.

LevitT, T. (1973). The Third Sector. New Tactics for a Responsive Society. Nueva York: A Division of American Management Associations.

MAYNTZ, R. (1999). «La teorie della governance: sfide e prospettive». Rivista Italiana di Scienza Politica, a. XXIX, no 1, p. 3-21.

NAVARRO, C.J. (1999). El sesgo participativo. Madrid: CSIC.

- (2001). La calidad del pluralismo del bienestar. Córdoba: Universidad de Córdoba. OfFe, C. (1990). Las contradicciones del Estado del bienestar. Madrid: Alianza.

PERRI 6 (1993). "Che cose e una associazione?». La Recerca Sociale, no 49, p. 37-58. Richardson, J.J. (1982). Policy Styles in Western Europe. Londres: George Allen \& Urwin.

Rodríguez Cabrero, G. (1994). «Estado del bienestar y sociedad de bienestar». Revista Internacional de Sociología, núm. 8 y 9, p. 7-29.

Rodríguez Cabrero, G.; Codorniú, M. (1996). «Las entidades voluntarias en la construcción del bienestar social». En RODRÍGUEz CABrero, G.; CODORNIÚ, M. (eds.). Las entidades voluntarias en España. Institucionalización, estructura económica y desarrollo asociativo. Madrid: MAS, p. 17-33.

SAlamon, L.; ANHEIER, H.K. (1992). "In search of the nonprofit sector I: the question of definitions». Working Paper $n^{\circ} 2$ of The John Hopkins Comparative Nonprofit Sector Project. [Publicado también en Voluntas, vol. 3, no 2 (1992), p. 125-152.] 
Salamon, L.; Hems, L.C.; Chinnock, K. (2000). «The Nonprofit Sector: For What and for Whom?». Working Papers of the Johns Hopkins Comparative Nonprofit Sector Project, no 37.

SARTORI, G. (1991). "Comparazione e metodo comparato». En SARTORI, G.; MorLINO, L. (eds.). La comparazione nelle scinze sociali. Bolonia: Il Mulino, p. 25-45.

SeIBEL, W.; ANHEIER, H.K. (1990). «Sociological and political science approaches to third sector». En ANHeIER, H.K.; SeIBel, W. The Third Sector. Berlín: Waler De Gruyter, p. 7-20.

Waterman, R.W.; Meier, K.J. (1998). «Principal-agent Models: An Expansion?». Journal of Public Administration Research and Theory, no 2, p. 173-202. 